\title{
La palabra, huésped y peregrina teatral
}

\author{
DIANA ACOSTA RIPPE
}

This article focuses on the staging of various theatrical and poetic works of Fernando Pessoa such as Fausto, O marinheiro (The Sailor) and the adaptation of the poem Antinous, to theatre. The article displays experiences of the analyses that were carried out for the staging of these three plays that are part of the theatrical way that Fernando Pessoa has been understood in Colombia and that, to a large extent, are known in the country in part thanks to the Colectivo Teatral Matacandelas from Medellin, Colombia. The text focuses on the drama em gente that unfolds from Pessoa's aesthetics to be performed in contemporary theatre, making the audience, the reader, the actor and the character endure different masks, different experiences of the static, the silent and of the word as the only inhabitant of the static body. In this text will be treated some personal experiences based on these three stagings.

THEATRE / DRAMA EM GENTE / INHABITANT WORD / STAGING / MATACANDELAS

El punto central de mi personalidad como artista es que soy un poeta dramático. Tengo, continuamente, en todo cuanto escribo, la exaltación íntima del poeta y la despersonalización del dramaturgo.

FERNANDO PESSOA

El heteronimismo siempre ha sido para mí una forma de teatro dentro de la literatura. Cuando conocí a Fernando Pessoa (y decir "conocer" es incurrir en una inexactitud porque habría que conocer a todos sus heterónimos, autores ficticios y personajes, para realmente conocerlo) supe que el drama no era la única manera en que la literatura y el teatro se entrecruzaban a varios niveles y en sus técnicas. He aprendido que el heteronimismo es quizá una de las maneras más originales de crear a un otro que se asemeja a un personaje teatral, pues labrar una vida 
vinculada a una obra, es una manera de dotar de realidad -de una casi de carne y hueso- lo que se escribe. Basta recordar a Federico García Lorca, quien, en una entrevista del 7 de abril de 1936 para $L a$ voz de Madrid, afirmó: "El teatro es poesía que se levanta del libro para hacerse humana". En el caso de Pessoa la poesía se hace persona, Pessoa es justamente un "drama en gente".

Cuando leí la versión de un Primer Fausto, publicada por el Fondo de Cultura Económica, surgió la posibilidad de llevar a la escena la adaptación de esta obra que, como casi todo en Pessoa, existía en los fragmentos que se conservan de ella. Debo decir que los entrenamientos físicos para llegar a la escena fueron más exhaustivos que los de una obra teatral convencional, pues pocas cosas son más exigentes que la quietud. Pessoa, aunque no había teorizado aún sobre el teatro estático, ya escribía obras en verso sin una acción móvil aparente, por ello, el montaje de una pieza detenida en el tiempo, o incluso sin tiempo, es todo un desafío. Tras el montaje surgieron nuevas ideas teatrales e incluso filosóficas sobre la imposibilidad que tenemos las personas de experimentar la quietud. Ahora bien, en mi intento por llevar a la escena esta obra, tuve que analizar al menos a dos personajes en la obra: a Fausto y a María, para entender por qué esas vidas están allí expuestas por Pessoa. El proceso para concebir las luces y una cierta atmósfera no fue fácil de lograr. Incluso la escenografía, que en este caso fue una mesa con papeles suspendidos en el aire que parecían caer y haberse detenido en el tiempo (quizá como la misma obra, o como Pessoa, en medio de versiones y contra versiones de sí mismo), fue la propuesta arriesgada: la mesa y sus papeles eran los únicos objetos que atenían los actores en la escena. En el caso de las luces, conviene aclarar que estas buscaban crear un juego entre la aparición y la desaparición constante de una serie de rostros - como si estos fueran algo móvil, al igual que la palabra, en medio de un universo estático-, un recurso, por lo demás, que aprendí del Teatro Matacandelas. Ese Primer Fausto buscó ser una aproximación al viaje interior de todo Fausto a través de la palabra, una palabra que debe ser leída pero que también puede ser actuada. La obra fáustica de Pessoa no tiene resolución, porque el personaje resulta ser como todo verbo hecho poesía, como agua que se filtra entre los dedos dándonos la sensación y el recuerdo de la humedad, pero no la promesa del agua.

El trabajo de pensar el Fausto fue extenso pues de Goethe viajé a Estanislao del Campo, de Marlowe a Calderón de la Barca, de Lord

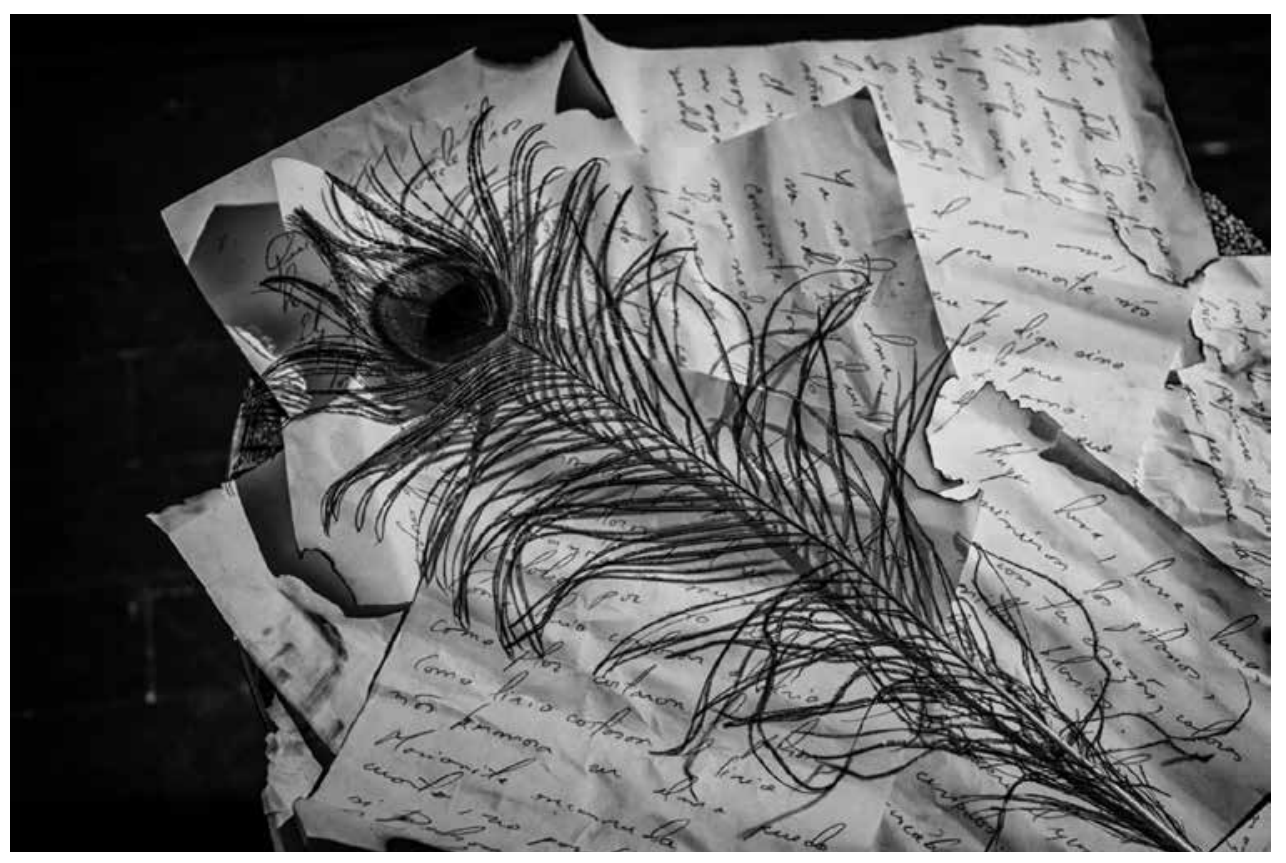

FAUSTO, DE FERNANDO PESSOA, ADAPTACIÓN DE DIANA ACOSTA RIPPE, BOGOTÁ, COLOMBIA, 2017, [F] MANUEL JOSÉ IBÁÑNEZ SILVA

Byron a Paul Valéry... Muchos autores me remitían de nuevo al mismo personaje, pero a medida que Fausto era más Faustos, el de Pessoa se me hacía más esquivo, si bien captaba mejor su diferencia, su profundidad. A la vez más inalcanzable, más interiorizado e incluso vital que alguien presente ante mí, el Fausto pessoano parecía burlarse ahora del teatro tras haber puesto en tela de juicio el conocimiento humano. Tal vez por este motivo, a la hora del montaje, los actos resultaban nulos, los dramas éramos a veces nosotros, los propios actores que, tras haber leído un aparente libreto, de repente caíamos en medio de un inabarcable "drama em gente".

El Fausto que dirigí y propuse se presentó en un pequeño teatro en la ciudad de Bogotá entre los años 2017 y 2018. También hubo una puesta en escena en el Cementerio Central de Bogotá, allí la acogida de la puesta fue masiva, casi parecía como si el entorno de la muerte hubiera contribuido para la atmósfera teatral, y para la mayor profundidad de la actuación. Los actores, como estatuas de piedra sobre la lápida del poeta colombiano José Asunción Silva, recitamos la obra pessoana como quien quiere traer a la vida a quienes yacen bajo una serie de lápidas medio olvidadas. 


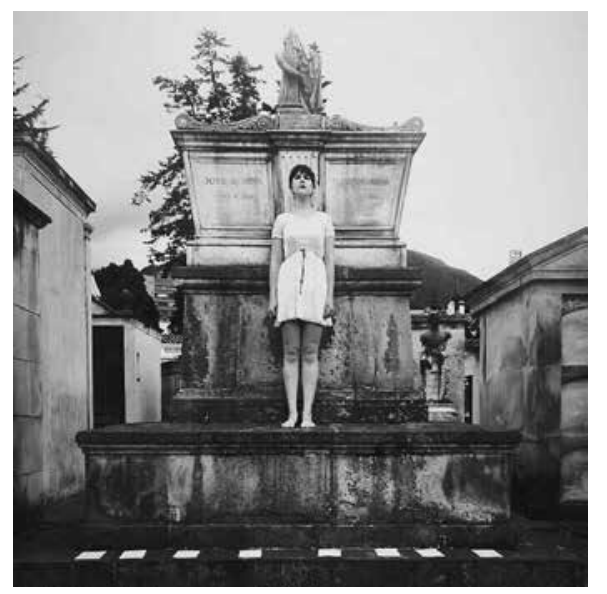

FAUSTO, DE FERNANDO PESSOA, ADAPTACIÓN DE DIANA ACOSTA RIPPE, CEMENTERIO CENTRAL DE BOGOTÁ, COLOMBIA, 2018, [F] MANUEL JOSÉ IBÁNEEZ SILVA

Sabemos que, entre el mes de enero y los primeros días de febrero de 1989, en la sala de teatro nacional Dona Maria II de Lisboa el actor y director portugués Ricardo Pais interpretó el papel de Fausto en una adaptación propia llamada Fausto, Fernando, Fragmentos, basándose en la edición trabajada durante años por la editora Teresa Sobral Cunha. A esta obra se suma la adaptación El desdoblamiento de Fausto, presentada y diseñada por António Lagarto, quien realizó una propuesta escénica y sonora con amplificación de distintas intensidades haciendo que la experiencia de partes de los textos del Fausto pessoano estuviera centrada en la sonoridad y la escenificación lumínicos. Entonces, varios actores interpretaron a Fausto y visualmente el montaje se escenificó a modo de historieta, es decir, de pequeñas cajas que contienen a un actor e imitando una cuadrícula. Unos años más tarde, Luis Miguel Cintra, un célebre actor portugués, realizó una lectura dramática del Primer Fausto, el 20 y 21 de noviembre del año 1997, en la ciudad de Madrid en el Teatro de la Abadía.

Obras como Fausto, en su carácter fragmentario, que va apareciendo a lo largo del tiempo, parecen corresponder a esa naturaleza ficticia del "drama em gente" en donde un ser va prefigurándose a través de las palabras y los tiempos, va armándose, va gestándose en el tiempo, en las ediciones, en sus distintas versiones de sí mismo.

En la misma expresión "drama en gente" se puede presentir -inscrita, cifrada - una posibilidad teatral que invoca a varias personas, que puede desbordar el texto escrito, que no alcanzan a abarcar las manos del personaje. El drama puesto en "gente" resulta esquivo al actor y al lector, invita a que convivamos sobre el mismo escenario, a que ejercitemos nuestra persona, que vivamos a Pessoa en ejercicio. El "drama en gente" se trata de una acción que sucede entre individuos. La palabra "drama" proviene del griego $\delta \rho \tilde{\alpha} \mu \alpha$ y traduce "hacer, ejecutar, efectuar, actuar". Un "drama en gente" supone inevitablemente un hacer, un ejercicio en personas, en gente: una acción que abarca a muchas personas y personalidades que hacen el ejercicio de ser y de dejar de ser. Es decir, el heteronimismo es la forma más precisa para entender el "drama en gente" pues Pessoa y los heterónimos conviven en un ejercicio poético donde son, conviven e incluso pueden dejar de ser, como en un teatro del lenguaje. Se sabe a cabalidad que Pessoa logró crear un drama ya no dividido en actos sino en gente, en otros. No en vano su heteronimismo es, justamente, eso: un "drama en gente".

Es curioso observar cómo este concepto deriva y proviene tanto de lo teatral como de lo poético, es decir, el "drama en gente" creado por Pessoa está construido en un ámbito poético pero su esencia es meramente teatral pues corresponde a un tipo de ejercicio de creación de personaje teatral, donde en el actor conviven diversos personajes y éste les presta su cuerpo para que ellos lo usen como un vehículo en donde conviven o han convivido muchos otros seres, otras gentes.

Es mejor no llover sobre mojado con relación al valor de los textos que Fernando Pessoa y sus heterónimos dejaron. Sabemos del arduo trabajo estético que estos revelan y de las múltiples exploraciones que implicaron. Pero aún así quedan muchas preguntas inquietantes por formular: ¿cuándo el actor, despojado de sí mismo, pasa a ser propiedad de sus personajes? ¿Cuándo, y en qué momento, Pessoa, es más, es menos o es equivalente a sus heterónimos? ¿El "drama em gente” podría verse como la coexistencia de todas las personas que conviven en un drama? ¿En uno de naturaleza poética? ¿El ortónimo, los heterónimos, el actor, el lector, el público son todos parte de un drama, de ese drama mayor?

El cuerpo del actor, como el del poeta es un albergue. En ambos casos, el lenguaje es "hóspede e peregrino" (Pessoa, 1990: t. 2, 110) ["huésped y peregrino"], para recuperar una expresión pessoana. La palabra no es un elemento ajeno o adicional a un rito, a un movimiento, sino un habitar; un espacio que habitamos y nos habita. Al fin y al cabo, el teatro en verso de Pessoa no se desarrolla solamente en el escenario, pues además hay que tener en cuenta la experiencia de la palabra poética. A veces parece como si el lector conviviera con el autor por un tiempo, como si sintiera la caricia de una brisa, como si el libro tuviera piel. Recordemos a Walt Whitman: "Camarada, esto no es un libro; quien toca esto toca a 
un hombre". El teatro y la poesía dramática de Pessoa (que es casi toda, incluso alguna más lírica en ocasiones) es otro rostro o máscara del teatro contemporáneo, y representa un lugar de quietud, de aliento en medio de la turbulencia móvil de otros semblantes teatrales y artísticos.

El poeta es un hospedaje de paso, así como el actor, pues ambos, de maneras distintas podrían ser asumidos como vehículos y recipientes del lenguaje. En el caso del poeta, éste podría ser un instrumento a través del cual el lenguaje poético fluye y se expresa a través de ese cuerpo instrumental, el poeta puede ser pensado como un vehículo a través del cual el lenguaje se filtra, se asienta y se expresa, usándolo como un médium, como un canal a través del cual la poesía circula haciéndolo escribir, cantar eso que el lenguaje necesita decir, o, mejor dicho, aquello que necesita ser escrito, ser expresado y, de paso, modifica para siempre la naturaleza sensible del o de la poeta. Inevitablemente cuando el lenguaje ha pasado a través de ese cuerpo, ese cuerpo no vuelve a sentir ni a pensar la poesía de la misma manera en la que lo hacía antes de haber sido vehículo de la misma. La palabra entonces es huésped y al mismo tiempo peregrina, va, se queda, se asienta, visita y luego vuelve a partir.

Ahora bien, en el caso del actor sucede que este puede ser también una guarida. Cuando tenemos un vaso que contiene agua lo llamamos, al menos en español, "vaso de agua" mientras que, cuando el mismo vaso contiene vino, el vaso cambia su manera de ser nombrado y se vuelve "vaso de vino". En el caso del actor, el ser biográfico tiene un nombre, pero, cuando interpreta corporalmente a un personaje literario y adopta su palabra, su vida, entonces es llamado como el nombre del personaje. El actor es un lugar en el que un personaje se aviva y habita como un huésped, por un tiempo, y para ello se ensaya para que se vaya incorporando hasta, que en algunos casos y a modo caníbal: el uno consume al otro. En el teatro la mudanza de palabra, de vida y de pensamiento se vive en la carne, el actor incorpora otra vida, es decir: otro lenguaje.

¿Cómo no citar la carta del 11 de diciembre de 1931 que Pessoa dirige a João Gaspar Simões? Dice Pessoa:

El punto central de mi personalidad como artista es que soy un poeta dramático. Tengo, continuamente, en todo cuanto escribo, la exaltación íntima del poeta y la despersonalización del dramaturgo [...] Desde que el crítico sepa, sin embargo, que soy en esencia poeta dramático, tendrá la llave de mi personalidad [...] Provisto de esta llave puede abrir lentamente todas las cerraduras de mi expresión [...] como poeta dramático me siento desprenderme de mí; que, como dramático (sin poeta), transmuto automáticamente lo que siento a una expresión ajena a lo que sentí, construyendo en la emoción a una persona inexistente que siento de verdad, y por eso siento, en derivación, otras emociones que yo, puramente yo, olvidé sentir. (Pessoa, 2015: 11)

El ser piensa cuando está en quietud. Ya Maurice Maeterlink y Chejov lo habían intuido tras abandonar la costumbre del gesto a veces inútil, del movimiento innecesario, para potenciar el surgimiento de una palabra en medio de un escenario detenido, porque la quietud es el silencio del movimiento. La quietud aguza la imaginación del lector y del espectador. No porque el teatro convencional, más móvil, o la danza misma no evoque las imágenes poéticas, sino porque el estatismo y el vacío contienen en sí la posibilidad de toda imagen, contribuyen a que la imaginación se erija y se despliegue. Para que una escena imaginaria vuele, sobra detener, aunque sea por un instante, la tiranía del movimiento. Antonin Artaud en su texto The Theatre and Its Double [El teatro y su doble], dice: "Destruir el lenguaje para alcanzar la vida es crear o recrear el teatro [...] lo importante es creer que no cualquiera puede hacerlo y que una preparación es necesaria”. (Artaud, 1969: 34). El trabajo con el lenguaje no es gratuito ni es una cuestión de pura genialidad; implica un profundo trabajo y preparación. Por su parte, el espectador debe construir una nueva experiencia estética, aventurándose por el reino de la quietud. La palabra, a través del cuerpo que la recibe (del actor, la actriz, el personaje) viva, crea y recrea incesantemente.

En Colombia, se tiene muy presente el avance editorial y de divulgación de la obra de Fernando Pessoa que ha logrado Jerónimo Pizarro. Si bien Pessoa era leído en Colombia desde décadas anteriores al propio montaje de $O$ marinheiro, realizado por el Teatro Matacandelas de Medellín desde el año 1990 (entidad a la cual muchas personas deben su primer encuentro con Pessoa en Colombia), y si bien el poeta dramático y algunos de sus heterónimos ya eran conocidos gracias a traductores y estudiosos tales como Ángel Crespo, Ángel Campos Pámpano, Teresa Rita Lopes y el mismo Eduardo Lourenço, lo cierto es que en la actualidad Jerónimo Pizarro es uno de los hombres más nombrados a la hora de citar a Fernando Pessoa, pues a él se debe que Pessoa haya sido editado y reeditado con muchísimo ahínco en el país. En ciudades como Medellín y Bogotá, los estudios y las ediciones de Pizarro son reconocidas y han facilitado la multiplicidad de lecturas de Pessoa, pues a sus estudios, 
ediciones y labores de coordinación se deben numerosas ediciones, traducciones y estudios. Sin duda hay diversos intelectuales que estudian y habitan el mundo a través de la estética de Pessoa, uno de ellos es Pizarro, otro Carlos Andrés Ciro, igualmente un fiel lector y traductor del poeta del "drama em gente" a quien también se asume como una fuente de consulta al tener una maravillosa "Pessoateca", una biblioteca repleta de Pessoa y sus heterónimos.

\section{ANTÍNOO, UNA NECESIDAD ESTÉTICA}

Antínoo (Antinous), siendo uno de los poemas que recrean ese Quinto Imperio espiritual que soñó Pessoa para convivencia y reunión de las almas, fue un poema llevado a la escena por el Colectivo Teatral Matacandelas de Medellín en octubre del 2019. La pandemia actual puso un alto en medio de su temporada, infelizmente. Antinous es un poema dramático. El texto, traducido al español, fue modificado en sus términos de los tiempos verbales para que, en la adaptación, el pasado transitara al presente, para que el lenguaje teatral apuntara a un "aquí y ahora". Esto no significa que la obra se haya "ambientado" para que pareciera de la actualidad, sino que su tiempo dramático se trajo al presente ajustando la voz del pasado. De hecho, la obra se transformó y se instaló en una idea de los días actuales para que Antínoo y el Emperador romano Adriano, en medio del esplendor de su mandato, coincidiera con el tiempo real de hoy de los espectadores. El teatro permite reunir dos o más tiempos en un solo espacio: uno puede remitir a la antigüedad, el otro a una determinada actualidad, por ejemplo; lo cual equivale a hacerle una finta a una idea del tiempo unívoca y cronológica.

Para recrear lo marmóreo, la palabra de ese amor, fue necesario entender que el cadáver de Antínoo no podría permanecer exclusivamente en la quietud, que tenía que estar en un mínimo movimiento -de cuerpo y de pensamiento-: transitando, así, de los gestos vitales hacia la quietud de la muerte. Si se quiere, la adaptación de Antínoo tiene algo de necromancia, en la medida en que revive al muerto que habla desde el mutismo de su cuerpo. Durante la adaptación de Antínoo (como lo tituló el colectivo teatral) no requería hacerse pasar del poema a las tablas, pero nosotros, como seres humanos, no podíamos no llevarlo a la escena porque se nos iría una posibilidad de condensar la vida y la experiencia del amor mismo, tan bellamente plasmado en el deseo de que lo amado se funda con el cosmos para luego convertirse en una constelación perpetua. Después de leer este poema, no ama uno. Del mismo modo y mucho menos, vuelve a pensar en el amor de la misma forma. Adaptar Antínoo de Pessoa fue una necesidad que tuvimos para entendernos mejor y entender de otra forma el amor. Por lo demás, bien sabemos que, por un texto de Pessoa, él no estaba interesado en juzgar el comportamiento de Adriano, amante del efebo Antínoo, sino de exorcizar, como poeta dramático, lo obsceno, esa categoría tan difícil de definir.

La adaptación de Antínoo fue posible gracias a la revisión de diversas traducciones del poema al español, pero siempre cotejando el texto original escrito por Pessoa en inglés y publicado en 1918. Se realizó una revisión de varias traducciones de Antínoo, entre las cuales: la traducción de Hernán Vargascarreño de 2015 (Ediciones Exilio), la de David Pujante y Carmen Torres de 2014; la de Cayetano Cantú y José Férez Kur de 2000; la de Luís A. Díez y José Luís Praga de 1995; la de Salustiano Masó de 1983. Por otro lado, se consultó el trabajo de Madeleine Jordá Billinghurst.

El montaje partió así de una primera fase de cotejo y estudio de diversas traducciones, proceso que derivarían en una "versión propia" realizada por el Colectivo Teatral Matacandelas. Antínoo, en tanto montaje teatral, se erigió sobre la selección de fragmentos que el colectivo consideró más bellos o acordes con su propuesta. En una segunda fase, la de llegar a una versión del colectivo, se designó a dos grupos de actores y actrices el estudio y memorización del texto para que ellos y ellas realizaran las primeras propuestas de puesta en escena, las cuales ya incluían voces dramáticas e interpretativas, música, coros, escenografía, vestuario e iluminación. Tras la presentación de esas diversas propuestas, el montaje alcanzó sus últimas fases, una de acoplamiento de las propuestas recibidas (aquellas de los dos grupos) y otra de finalización, para preparar una obra ya más completa para los primeros ensayos. La obra se fue desarrollando a medida que transcurría el montaje pues, durante el mismo se surgieron nuevas propuestas e se hicieron ajustes.

Antínoo encarna el amor y la muerte, Adriano encarna el amor y el deseo de la eternidad, de la memoria que busca prevalecer, del amor que no se extingue del cuerpo del amante, de las estatuas erigidas en honor de un recuerdo que no se quiere muerto. Esta obra de Pessoa no es ajena al estado de la quietud, en el cual el poema parece inmerso. En la adaptación hay movimiento, pero lento, con cadencia de quietud, de movimiento ritual, de pausa para soñar. 


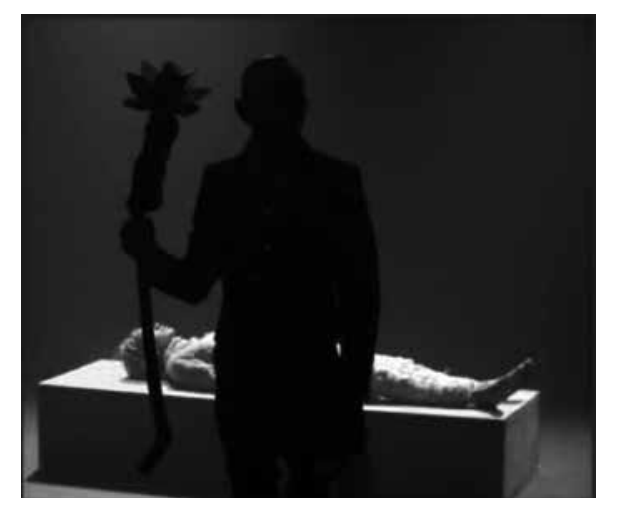

ANTÍNOO, DE FERNANDO PESSOA, ADAPTACIÓN COLECTIVO TEATRAL MATACANDELAS, MEDELLİ́n, COLOMBIA, 2019 , [F] ARCHIVO MATACANDELAS

En Antínoo hay una evocación del amor homosexual, pero definir la obra como un poema homoerótico sería reduccionista. La propuesta estética de Pessoa abarca muchos aspectos y se preocupa por cuestiones formales como, por ejemplo, la de cómo integrar tópicos helenísticos, romanos y egipcios en un texto sobre la muerte y el amor. Además de recuperar un mito, hay una honda preocupación por la forma y el lenguaje del poema. En Antínoo importan el amor y la pérdida, la muerte y lo eterno.

En el montaje teatral del Matacandelas el personaje de Antínoo y los dioses Júpiter, Hebe y Ganimedes permanecen fijos, pero los mortales aún vivos, es decir, el coro de habitantes del imperio de Adriano y el mismo Emperador se mantienen móviles, como si, simbólicamente, lo amado y lo elevado fueran lo fijo y lo vivo fuera lo móvil. El Antínoo del Matacandelas facilita dos experiencias: de lo amado a través de lo estático y aquella de lo vivo en lo dinámico. Adriano, aunque ha perdido a su amado, quiere que sea recordado para siempre. Hay una tensión entre el reposo y la acción.

En el caso de Cármides, personaje de un diálogo platónico, homónimos, y en el caso de Antínoo, la belleza del joven es narrada a través de las palabras de sus admiradores. Detrás de ambas historias hay una serie de versiones y referencias no siempre coincidentes. Durante la preparación del montaje teatral de Antínoo, que implicó un arduo estudio, a la vez histórico y mitológico, se redescubrieron, por ejemplo, diferentes mitos relacionados con la muerte de Antínoo. Uno de los más citados afirma que Antínoo (ejerciendo una de las formas sacrificiales griegas en honor a un gobernante) se arrojó al río Nilo el 30 de octubre del año 130 d.C., acaso para atraer así, mediante un sacrificio ritual, el favor de los dioses para el Emperador Adriano. Esta versión fue decisiva en la adaptación de la obra pessoana por el colectivo Matacandelas. "Antínoo se arrojó a las aguas del Nilo, sacrificándose por su emperador". (Fernández/ /Pordomingo, 2017: 216). En otra versión del mito de Antínoo, surge el tópico latino de la metamorfosis: al parecer, Antínoo, convertido en flor de loto roja. Su nombre y su persona se identificarían con una flor color rojo sangre. Según el poeta clásico Páncrates de Alejandría (contemporáneo de Adriano), el Emperador dio muerte a un león con su jabalina para evitar que éste atacara a Antínoo y entonces, en la arena, donde cayó la sangre del león, surgió "la flor de Antínoo", que se llamó "Antinoeios" o "Antinoeia", que encarnaría la flor que nace de la sangre de un animal sacrificado para salvar la vida de un efebo. Es por esta segunda versión del mito que, al inicio de la obra montada en Medellín, el personaje que parece ser un guía, carga una flor de loto roja.

Y Páncrates, un poeta de allí que nosotros conocimos, al emperador Hadriano, cuando visitó Alejandría, le mostró como un gran milagro el loto rojo, diciendo que había de llamarlo de Antínoo, porque surgió de la tierra cuando recibió la sangre del león mauritano, al que Hadriano en una cacería en Libia, en las cercanías de Alejandría, había dado muerte [...] y Páncrates en el poema dice, no sin elegancia: "El tomillo rastrero, el blanco lilis o el Jacinto de color púrpura o las flores de la azul celidonia y la rosa que se abre a los céfiros de la primavera: pero jamás antes había florecido la flor de Antínoo". (Fernández/Pordomingo, 2017: 216-217)

Otros datos fueron también definiendo rasgos y perfiles de la obra. Así, por ejemplo, descubrimos que la parte sur de la constelación de Aquila se llamó Antínoo y que no era imposible que así la hubiera designado Adriano en memoria del amante ahogado en el río Nilo. A causa de esta información, existe una escena en la cual se recrea la constelación de Antínoo mediante juegos de luces, casi como un planetario.

Antínoo nos instala en medio de la galaxia del "drama em gente", nos permite adentrarnos en imperios tan antiguos como el amor mismo y nos traslada, a través de la belleza de la palabra poética, a la profundidad del cielo en sus infinitas constelaciones. Entre el cielo y el Nilo, entre la tumba y el beso de los labios fríos, entre el epitafio cantado y el silencio del aceite más perfumado, trascurre la historia de Antínoo adaptada por Matacandelas, pionero, en el mundo del teatro, en la adaptación del Antínoo pessoano. 
Antínoo es un poema, pero fue leído y asumido como un drama y esta forma diferente de asumirlo hizo que fuera más visible la dimensión dramática de la obra y que las palabras fueran moduladas de otras formas por nosotros, los actores, huéspedes y peregrinos de las palabras del autor.

\section{EL MARINERO Y EL TEATRO MATACANDELAS}

El Teatro Matacandelas se define a sí mismo como una pandilla que actúa como una institución. No es gratuito el verbo elegido "actuar" pues se trata de uno de los grupos más importantes en el área teatral y escénica en Colombia. El Colectivo Teatral Matacandelas no es solamente un grupo de teatro, es una patria independiente que trata de tener buenas relaciones políticas con Colombia, a quien denomina "un país vecino". Fue fundado en el año 1979 en la ciudad de Envigado, en el departamento de Antioquia para unos años más tarde transitar a la capital de este departamento: Medellín, donde hoy se mantiene presente.

Matacandelas es mundialmente reconocido por varios de sus montajes teatrales, entre los cuales está $O$ marinheiro [Elmarinero] de Fernando Pessoa, The Girl Who Wanted to be God [La chica que quería ser Dios] obra creada y adaptada a través de los textos de la poeta Sylvia Plath, Angelitos empantanados de Andrés Caicedo, escritor y dramaturgo colombiano, Fernando González: velada metafísica, una obra basada en las libretas y textos del filósofo antioqueño Fernando González Ochoa.

Matacandelas es un colectivo que vive democráticamente de, por y para el teatro. Como decía Diego Sánchez, codirector y actor de Matacandelas y a quien la muerte ocultó en el año 2018: "Como no nos gusta este país en el que vivimos, nos inventamos otro que se llama Matacandelas. Como no nos gusta mucho este mundo que nos han creado, creamos otros en el escenario".

La obra $O$ marinheiro [El marinero] se conoció en Colombia, en gran medida, gracias a la adaptación hecha por el Colectivo Teatral Matacandelas de Medellín. La obra fue estrenada a finales de 1990 en medio de una ciudad sacudida por la violencia de unos narcotraficantes que desafiaron al Estado, y de otros muchos agentes violentos (sin olvidar otras violencias más veladas y menos espectaculares). El colectivo decidió respetar el título en portugués y por ello en Colombia se ha conocido la obra como O marinheiro más que como "El marinero". Pasados treinta
O MARINHEIRO, DE FERNANDO PESSOA, ADAPTACIÓN COLECTIVO TEATRAL MATACANDELAS, MEDELLIN, COLOMBIA, 2015 [F] ARCHIVO MATACANDELAS

años, la propuesta sigue siendo ensayada y representada. La propuesta se caracteriza por la creación de una atmósfera de serenidad solemne, en medio de la cual las veladoras - con ojos profundos y negros, con sus vestimentas negras y un maquillaje que da la impresión de que llevan máscaras blanca - se mantienen como suspendidas en un mundo soñado, en un mundo casi vaporoso y enigmático. La pieza, en un acto estático, chocó a algunos espectadores que exigían que el teatro "respondiera" a una realidad convulsionada, ya fuera mostrando otra igual de agitada o evitando un distanciamiento poético como el de una obra simbolista. Pero el Matacandelas no creyó que tuviera que buscar otra obra diferente a $O$ marinheiro, ni que tuviera que volver sangrienta la pudorosa obra pessoana. La adaptación teatral no buscaba ser una contraparte a la violencia, sino, más bien, un respiro, un paréntesis en medio de la violencia. Luego de un inicio difícil, pues la recepción no fue la mejor, $O$ marinheiro triunfó y se volvió una de las obras de mayor éxito en el teatro colombiano. A lo largo de las tres décadas, $O$ marinheiro ha sido un montaje masivamente acogido por el público nacional. Es como si cada espectador quedara atado a las palabras de Pessoa gracias a las voces de tres deslumbrantes actrices que habitan transitoriamente esas palabras. Si en la lectura la quietud tiene un eco, en la representación, en una sala de teatro, aún más, pues en medio de la cueva donde está el tablado del sobrecogimiento y la quietud, estos son aún mayores y compartidos por más personas.

Cristóbal Peláez, el director del colectivo Matacandelas recuerda vivamente una ocasión en la que el público, en la primera presentación de la obra, permaneció diez minutos mudo en la sala, sin reacción, sin aplausos, como si el estatismo y la sensación de estar viviendo un sueño los hubiera consumido, imbuido en la escena. Los canapés y las bebidas 
de coctel listas para sus primeros invitados quedaron intactas pues el público (invitados a esa primera presentación en noviembre de 1990) se marchó sin probar bocado e ignorando el convivio posterior. Exacto o no, este relato es el de la estupefacción que $O$ marinheiro deja en quien lo lee o asiste a verlo puesto en escena. Estático puede quedar un público que por dentro aún se agita y no sabe adónde ha entrado y si ya salió, al final, de ese lugar no localizado.

Con el pasar del tiempo y de innúmeras presentaciones, $O$ marinheiro del Matacandelas se convirtió en una obra de culto a la que un público cada vez más cautivo acudiría, como si de un culto se tratara. Muy pronto, los espectadores de la obra supieron que era banal exigir que la obra dialogara directamente con la realidad del país, entre otras cosas porque tal vez sí lo hacía; a fin de cuentas, $O$ marinheiro es la historia de un duelo y de una velación. Sólo no lo veía quien esperaba algo menos sutil y poético.

Sin querer desconocer otras propuestas que se han realizado con esta obra a lo largo del tiempo -en otras ciudades, con otros medios y sobre otros escenarios teatrales- debo decir, con todo, que a $O$ marinheiro del Matacandelas se debe el hecho de que Pessoa sea aún más aclamado, encarnado, pensado y conocido en esta ciudad de Medellín y en Colombia, en general. La obra con su ambientación escueta, con sus árboles secos, con su iluminación misteriosa, con los rostros silentes de esas veladoras-veladas nos transporta a un mundo y una palabra que tienen el nombre de una revelación: Pessoa. El marinero es una obra de tránsito en medio de la quietud, en la que las tres veladoras inmóviles, como posibles emanaciones de las primeras horas del día, como aparentes desdoblamientos de una trinidad, de una única palabra, se presentan como cuerpos cercados por el sueño y un cierto spleen. Se trata además de una obra que suspende la realidad, o nuestra creencia o descreencia de ella, pues a lo largo de su lectura o audiencia dudamos de estar despiertos, así como de haber alguna vez despertado.

\section{REFERENCIAS BIBLIOGRÁFICAS}

ARTAUD, Antonin (1969), El teatro y su doble, trad. Enrique Alonso y Francisco Abelenda, La Habana, Editorial del Instituto del libro.

FERNÁNDEZ, Delgado José Antonio, e PORDOMINGO, Francisca (2017), La retórica escolar griega y su influencia literaria, Salamanca, Ediciones Universidad de Salamanca.

GARCÍA LORCA, Federico (1936), "Entrevista", in La voz de Madrid. Consulta el día 9 de abril de 2020, https:// belendo.wordpress.com/2008/02/17/lorca-entrevista-en-la-voz-de-madrid-el-7-de-abril-de-1936/.

PESSOA, Fernando (2016), Fausto. Pessoa Múltiple, Antología Bilingüe, Bogotá, Edición Fondo de Cultura Económica.

- (2015a), «Antínoo», Revista Exilio, n. 25, Mayo.

- (2015b) El marinero, trad. Nicolás Barbosa López, Medellín, Editorial Tragaluz.

- (2015c) El marinero, “Carta a João Gaspar Simões", trad. Nicolás Barbosa López, Medellín, Editorial Tragaluz.

- (2014), Antinoo, trad. David Pujante, Carmen Torres, Lisboa, Editorial Salto de página.

- (1990) Pessoa por Conhecer - Textos para um novo mapa, edição de Teresa Rita Lopes, Lisboa, Estampa.

PLATón (2017), Cármides, trad. Jorge Torres De La Cerda, Santiago de Chile, Editorial Universitaria.

WHITMAN, Walt (2014), Hojas de hierba, traducción, introducción y notas Eduardo Moga, Barcelona, Editorial Galáxia Gutemberg-Círculo de lectores.

\section{DIANA ACOSTA RIPPE}

-

Licenciada em Estudos Literários pela Pontificia Universidad Javeriana de Bogotá, na Colômbia, e Mestre em Teatro e Artes Vivas da Universidad Nacional de Colombia, exerce funções como directora e actriz de teatro. Levou à cena obras de Fernando Pessoa, Samuel Beckett, Ovídio, Sófocles, Ésquilo e Eurípides. É ainda consultora literária em encenações teatrais e professora de oficinas de literatura, línguas e teatro. É afiliada dà Universidad Javeriana. 